\title{
A Pilot Study of the Effect of Outsole Hardness on Lower Limb Kinematics and Kinetics during Soccer Related Movements
}

\author{
by \\ Dong Sun ${ }^{1}$, Qichang Mei ${ }^{1,2}$, Julien S Baker ${ }^{3}$, Xuewen Jia ${ }^{4}$, Yaodong $G u^{1,2}$
}

The purpose of this study was to investigate the effect of different outsole hardness of turf cleats shoes on the lower limb kinematics and kinetics of soccer players playing on artificial turf. The participants were required to complete tasks of straight running and $45^{\circ}$ left sidestep cutting movements, respectively, at the speed of $4.5 \pm 0.2 \mathrm{~m} / \mathrm{s}$ on artificial turf. They were asked to randomly select turf cleats shoes with a soft outsole (SO), medium hardness outsole (MO) and hard outsole (HO). During the stance phase of straight running, peak pressure and force-time integral in medial forefoot (MFF) of players wearing cleats shoes with MO were significantly higher than those wearing cleats shoes with SO. During the stance phase of a $45^{\circ}$ cutting maneuver, players wearing cleats shoes with SO showed significantly higher peak knee flexion and abduction angles than the HO group. Players wearing cleats shoes with SO also showed higher ankle dorsiflexion and inversion angles compared with those wearing cleats shoes with HO. The vertical average loading rate (VALR) as well as peak pressure and force-time integral in the heel $(H)$ and lateral forefoot (LFF) regions of players wearing cleats shoes with $\mathrm{HO}$ were significantly higher than those wearing shoes with SO. On the contrary, peak pressure and force-time integral of players wearing shoes with SO were significantly higher than those wearing shoes with HO in MFF. A higher vertical loading rate and plantar pressure of some areas may increase the potential risk of metatarsal stress fractures and plantar fasciitis. Therefore, this finding about turf cleats shoes could give some theoretic support for the design of turf cleats shoes and material optimization in the future.

Key words: turf cleats shoes; outsole hardness; performance; injury.

\section{Introduction}

Soccer is the most popular sport all over the world. According to statistics from FIFA (Federation Internationale de Football Association), the number of professional and amateur soccer players is about 270 million spread around more than 200 countries and regions in the world. Soccer can be played on a variety of surfaces, such as natural grass, artificial turf, indoor wood and synthetic floors. In recent years, with the popularity of artificial turf, turf cleats shoes (short cleats covering the entire sole) have been found having better impact absorption compared with longer and aggressive studs shoes on artificial turf (Matsuo et al., 2011). Athletic shoes are support surface interfaces composed of relatively soft compressible materials designed to protect against sports injuries through decreasing vertical impact. Appropriate functional footwear could effectively attenuate impact forces generated by foot contact with the ground during various impact activities (Nigg et al., 1995).

The cushioning properties of shoes have been stated to be an important regulator of footwear comfort (Nigg et al., 2012). High correlations have been found between cushioning perception and biomechanical variables in shoes

1 - Faculty of Sports Science, Ningbo University, China.

2 - Research Academy of Grand Health Interdisciplinary, Ningbo University, China.

3 - School of Science and Sport, University of the West of Scotland, UK.

4 - Department of Orthopaedics, Ningbo First Hospital, China. 
of different sole hardness (Hennig, 2011). Sole hardness can influence runners' functional characteristics, subsequently altering loading patterns and foot motion. Variations in the hardness of the shoe sole may therefore influence both kinematics of lower limbs and the magnitude of loading under the foot. Kinematics and kinetics are highly associated with non-contact injuries of lower extremities, player's comfort and performance. It is generally assumed that high impact forces and over pronation are connected with pain and injuries in running. During running and cutting movements, some plantar regions may experience repeated impact force between the foot and the sole surface (Morio et al., 2009). Sport shoes manufacturers have designed a variety of shoes with various outsole materials to improve cushioning properties, prevent noncontact injuries of lower extremities and enhance athletic performance. One of the most important goals in designing sport footwear is to reduce the force peaks (Nigg, 2012).

During running, sprinting and cutting movements, large differences in plantar pressure patterns have been found. Elis (2004) and Wong et al. (2007) reported higher medial forefoot pressure during sprinting and cutting movements. It was also speculated that high medial forefoot loads in sprinting and cutting movements could be the reason for the high incidence of metatarsal stress fractures of soccer players (Wong et al., 2007). These different loading patterns may explain some of the overuse injuries in soccer (Henning, 2014). A stress fracture is a common sports injury. During soccer-related movements, some plantar regions could bear double or triple body weight. The additional pressure of these specific plantar regions may lead to potential risks of plantar fasciitis or metatarsal stress fractures (Lieberman et al., 2000). Sole hardness may influence bending stiffness of shoes, as well as biomechanical characteristics of lower limbs during running. Sakaguchi et al. (2015) stated that performance may in fact increase with increased bending stiffness of the shoe.

Sport footwear consists of several components including a midsole, outsole, heel counter and upper. Of these, outsole hardness has been predominantly investigated on running and walking to optimize footwear cushioning properties (Nigg et al., 1987; Sterzing et al., 2013).
However, only few studies focused on the outsole hardness considering performance and noncontact injuries in soccer. Thus, the objective of this study was to investigate the effect of different outsole hardness on the lower limb kinematics and kinetics in soccer players playing on artificial turf.

\section{Material and Methods}

\section{Participants}

The study was approved by the local ethics committee of the Ningbo University. Prior to the experiment, the subjects were informed of the objectives of the study and experimental procedures. All subjects provided informed written consent to participate in the study. Sixteen male soccer players (age: $20.2 \pm 1.4$ years, body height: $171.5 \pm 5.3 \mathrm{~cm}$, body mass: $65.3 \pm 5.6 \mathrm{~kg}$, soccer experience: $11.3 \pm 3.1$ years) from the university soccer team were recruited for this study. The inclusion criteria were: dominance of the right leg, and to be free of major injuries to the lower extremities for the past 6 months.

\section{Experiment shoes}

The turf shoes with different outsole hardness were provided by the ANTA Sports Science Laboratory. The outsoles were all made of synthetic rubber, except hardness, the design of the outsole was exactly the same (Table 1). The Elastic modulus (E, MPa) of outsole materials were measured with a material hardness test machine (Instron AG Grove USA) (Li et al., 2015). The hardness of outsole materials is indicated with Shore A Hardness (HA, ${ }^{\circ}$ ), which is generally used to measure the hardness of rubber products; the greater its value, the higher the hardness (Zhao et al., 2015). The equation of Elastic modulus (E) and outsole hardness (HA) is as follows (Wang et al., 2011):

$$
\mathrm{E}=(15.75+2.15 H A) /(100-H A)
$$

Elastic modulus of the three outsole materials calculated from the equation above were 5.92, 7.21 and 9.68. Shore A Hardness (HA) values of the three outsole materials were $71.4^{\circ}, 75.3^{\circ}$ and $80.5^{\circ}$, indicating soft outsole (SO), medium hardness outsole (MO) and hard outsole (HO), respectively (Table 1).

\section{Procedures}

All running tests and experiments were 
conducted in the Sports Biomechanics Laboratory of the Ningbo University. An eight-camera Vicon motion analysis system (Oxford Metrics Ltd., Oxford, UK) was used to capture participants' lower limb kinematics at the frequency of $200 \mathrm{~Hz}$. A plug-in-gait model marker set was used in this study. Subjects were required to wear tight-fitting pants and 16 reflective markers (diameter: $14 \mathrm{~mm}$ ) were attached with adhesive to left and right lower limbs respectively on different key locations including the anterior-superior iliac spine, posterior-superior iliac spine, lateral mid-thigh, lateral knee, lateral mid-shank, lateral malleolus, second metatarsal head and calcaneus. The marked points on the second metatarsal head and calcaneus were placed on the corresponding anatomical position of shoes. The stance phase was defined as the heel of the right foot contact ground to the toe off the ground, kinematic variables were the peak and mean (standard deviation) values of each joint-angle (ankle, knee and hip) during the stance phase.

The in-shoe plantar pressure measurement system (Novel Pedar System, Germany) was used to measure the pressure and force exerted on the insole pressure sensors, which were divided into seven anatomical parts, including the heel $(\mathrm{H})$, medial foot (MF), medial forefoot (MFF), central forefoot (CFF), lateral forefoot (LFF), big toes (BT) and other toes (OT) (Figure 1). All insoles used in the experiment had been regulated with a pressure pump before each participant's test. All subjects ran with the right foot step onto the force plate (Kistler, Switzerland), which was fixed $6 \mathrm{~m}$ away from the starting line and utilized to collect kinetic data at the frequency of $1000 \mathrm{~Hz}$. The height of the artificial turf fiber was $40 \mathrm{~mm}$ without any infill or a shock pad, to ensure accuracy of kinetic data collection. A separate piece of artificial turf was cut to $60 \times 90 \mathrm{~cm}$ to fit the dimension of the force plate and fastened by double-sided tape. The design of the experiment protocol is presented in Figure 2.

A 3 min warm-up before the experiment for each subject was allowed, shoe order and movements were randomized across subjects. Both straight-ahead running and $45^{\circ}$ sidestep cutting were performed at the speed of $5.0 \pm$ $0.2 \mathrm{~m} / \mathrm{s}$. Velocity of straight-ahead running and cutting movements was measured using Brower timing lights (Brower Timing System, Draper, UT,
USA), positioned $1.2 \mathrm{~m}$ apart, centering the force plate. Participants warmed up and familiarized themselves with the laboratory environment and target speed prior to data collection. Subjects were given $1 \mathrm{~min}$ rest between trials and $5 \mathrm{~min}$ rest between shoe and movement conditions. If the subject did not land with the right foot on the force plate, the trials would be discarded and the subject would be asked to repeat this movement. Subjects were asked to land near the center of the force plate to ensure accurate force collection. Subjects were instructed to perform heel landing of cutting movement, and without requirement for the landing pattern of straight-ahead running. Six successful trials were conducted under each condition, with synchronized collection of kinematic and kinetic data.

\section{Statistical analyses}

The SPSS 17.0 software (SPSS Inc., Chicago, IL, USA) was used for statistical analysis. The Post Hoc Multiple Comparisons were performed and LSD (least significance difference) of one-way ANOVA (analysis of variance) was taken for kinematics, variables of ground reaction force, peak pressure and force time integral of straight running as well as $45^{\circ}$ cutting movement. The level of significance was set at 0.05 .

\section{Results}

Three dimensional kinematics of knee and ankle joints were analyzed during the stance phase of $45^{\circ}$ cut. Ankle and knee kinematic variables in different shoe conditions are presented in Picture 1. In the sagittal plane, the peak ankle dorsiflexion angle wearing shoes with SO was significantly higher than when wearing shoes with HO $(p<0.01)$, the peak knee flexion angle wearing shoes with $\mathrm{SO}$ was significantly higher than when wearing shoes with $\mathrm{MO}(p=$ $0.017)$ and $\mathrm{HO}(p=0.013)$. In the coronal plane, the peak knee abduction angle wearing shoes with $\mathrm{SO}$ was significantly greater than when wearing shoes with HO $(p=0.016)$. The peak ankle inversion angle wearing shoes with $\mathrm{SO}$ was significantly greater than when wearing shoes with $\mathrm{HO}(p=0.032)$. No significant difference was observed in the hip joint in sagittal, coronal and transverse planes.

Ground reaction forces (GRF) of each subject were normalized to body weight (BW), and peak vertical ground reaction force (VGRF) showed no 
significant difference between the three hardness conditions. Although, the vertical average loading rate (VALR) when wearing shoes with $\mathrm{HO}$ was significantly higher than when wearing shoes with SO in the stance phase of $45^{\circ}$ cutting movement. The stance phase of $45^{\circ}$ cut can be divided into weight acceptance, peak push-off and final push-off. The impact force during running has two distinct peaks, the first peak is the impact peak and the second one is the active peak (Picture 2).

Due to different foot strike patterns of straight running, the comparative analysis of different hardness shoes was only performed on the forefoot and toes. During the stance phase of straight running, peak pressure and force-time integral in the MFF region wearing shoes with MO were significantly higher than when wearing shoes with SO. During the stance phase of $45^{\circ} \mathrm{cut}$, plantar pressure showed significant differences between different shoe conditions in the heel $(\mathrm{H})$, MFF and LFF foot regions. Peak pressure and force-time integral of players' wearing shoes with HO showed significantly higher values than when wearing shoes with SO in the heel and LFF regions. However, in the MFF region, peak pressure and force-time integral when wearing shoes with SO were significantly higher than when wearing shoes with $\mathrm{HO}$ (Picture 3).

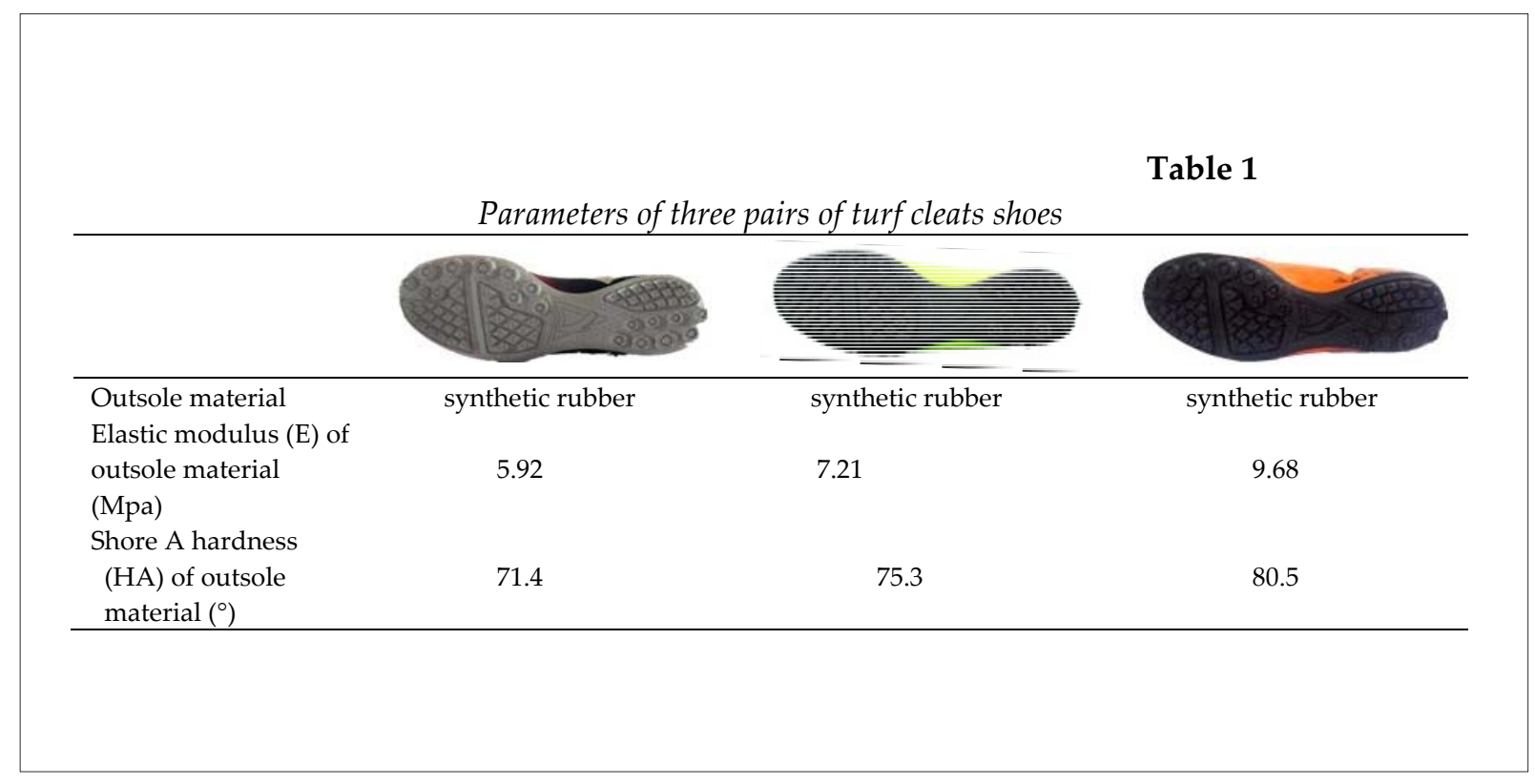

\begin{tabular}{|c|c|c|c|c|c|c|}
\hline \multicolumn{7}{|c|}{ Variables of Ground Reaction Force, mean $(S D), N=16^{\text {Table } 2}$} \\
\hline & \multicolumn{3}{|c|}{ straight-ahead running } & \multicolumn{3}{|c|}{$45^{\circ}$ sidestep cutting } \\
\hline & SO & $\mathrm{MO}$ & $\mathrm{HO}$ & SO & $\mathrm{MO}$ & $\mathrm{HO}$ \\
\hline Contact time (s) & $\begin{array}{c}0.166 \\
(0.045)\end{array}$ & $\begin{array}{c}0.164 \\
(0.025)\end{array}$ & $\begin{array}{c}0.163 \\
(0.037)\end{array}$ & $\begin{array}{c}0.213 \\
(0.016)\end{array}$ & $\begin{array}{c}0.210 \\
(0.008)\end{array}$ & $\begin{array}{c}0.209 \\
(0.013)\end{array}$ \\
\hline $\begin{array}{l}\text { Peak vertical ground } \\
\text { reaction force }(\mathrm{BW})\end{array}$ & $\begin{array}{l}2.57 \\
(0.17)\end{array}$ & $\begin{array}{c}2.60 \\
(0.27)\end{array}$ & $\begin{array}{l}2.58 \\
(0.29)\end{array}$ & $\begin{array}{c}2.77 \\
(0.033)\end{array}$ & $\begin{array}{c}2.75 \\
(0.023)\end{array}$ & $\begin{array}{c}2.74 \\
(0.026)\end{array}$ \\
\hline $\begin{array}{l}\text { Vertical average loading } \\
\text { rate }\left(\mathrm{BW} \cdot \mathrm{s}^{-1}\right)\end{array}$ & 1 & 1 & 1 & $\begin{array}{l}90.3 \\
(6.7)^{*}\end{array}$ & $\begin{array}{l}92.4 \\
(5.8)\end{array}$ & $\begin{array}{l}94.5 \\
(7.1)\end{array}$ \\
\hline \multicolumn{7}{|c|}{$\begin{array}{c}B W=\text { body weight; } S O=\text { soft outsole; } M O=\text { medium hardness outsole; } H O=\text { hard outsole. } \\
* \text { Significant difference between } S O \text { and HO turf shoes, } p<0.05 .\end{array}$} \\
\hline
\end{tabular}



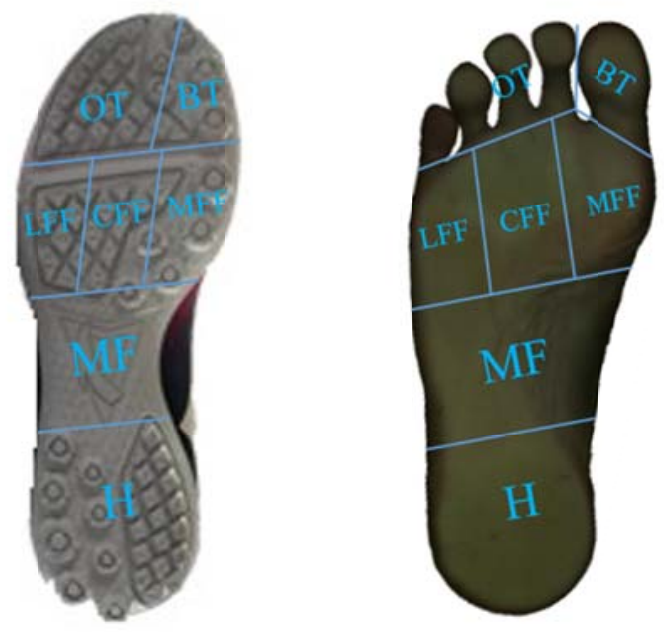

Figure 1

Anatomical areas of the plantar region

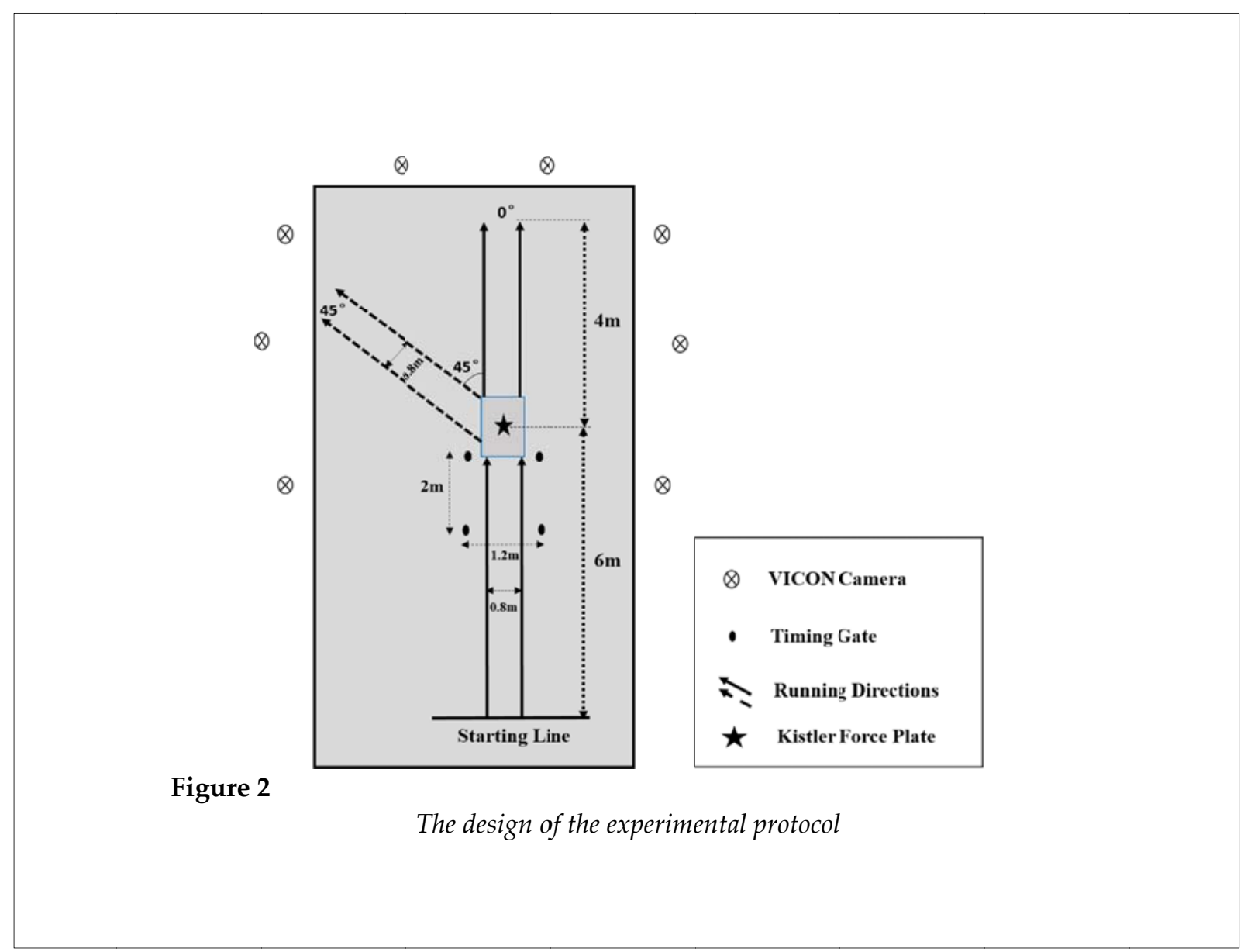

(c) Editorial Committee of Journal of Human Kinetics 
A

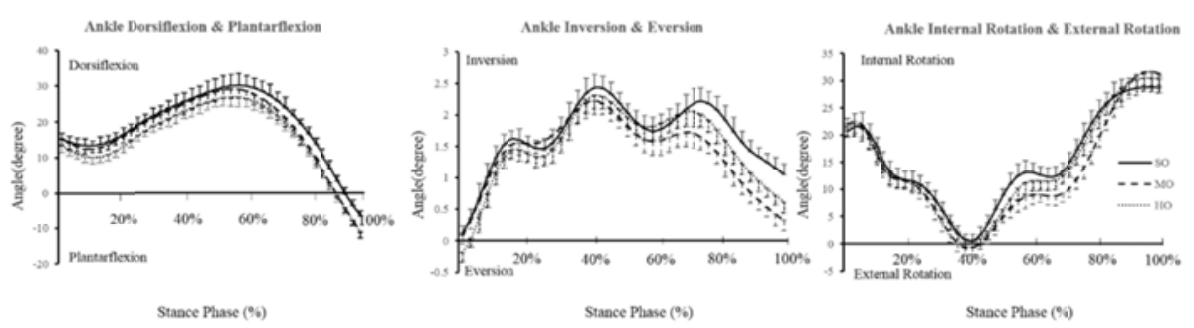

B
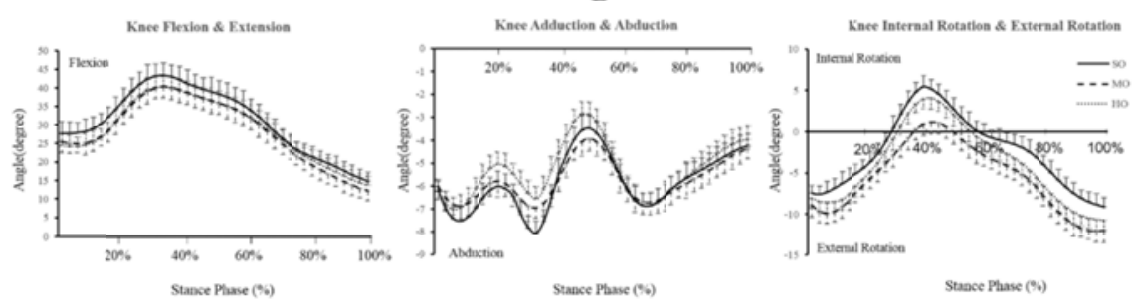

$\mathrm{C}$
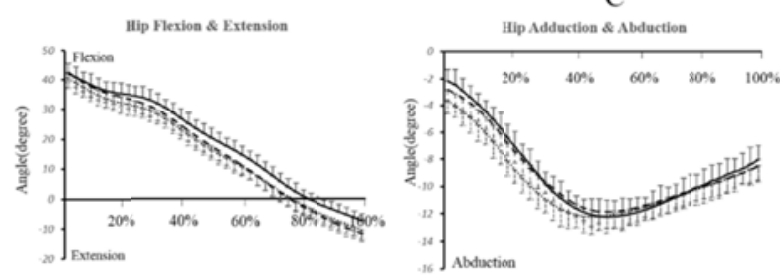

Stance Plase (\%)

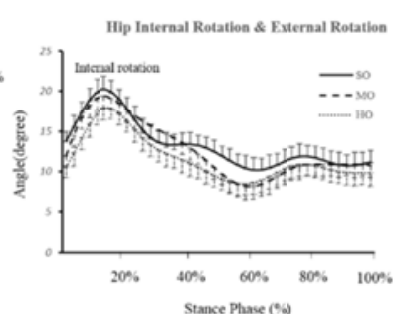

Picture 1

$A, B$ and $C$ represent ankle, knee and hip angle curves in the stance phase of $45^{\circ} \mathrm{cut}$, respectively

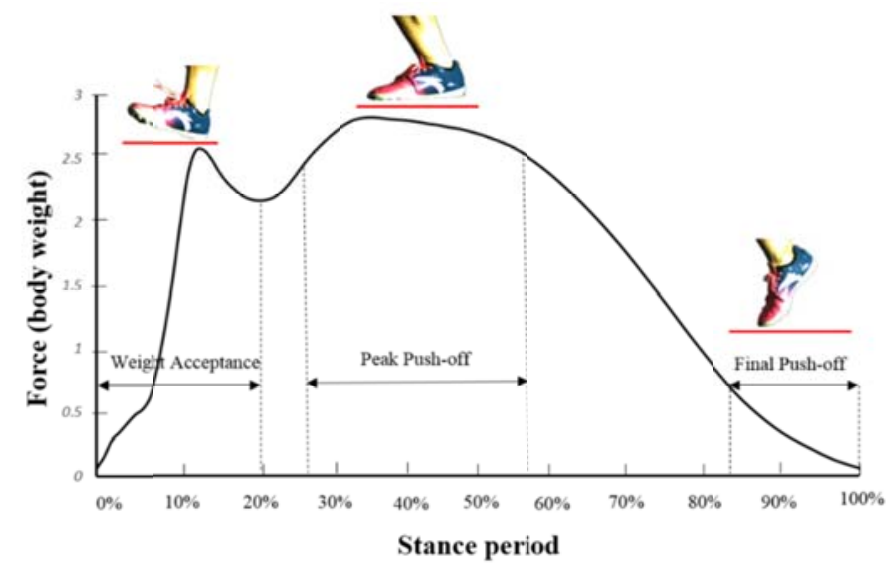

Picture 2

Vertical ground reaction force during the stance period of $45^{\circ} \mathrm{cut}$ when wearing SO turf shoes 


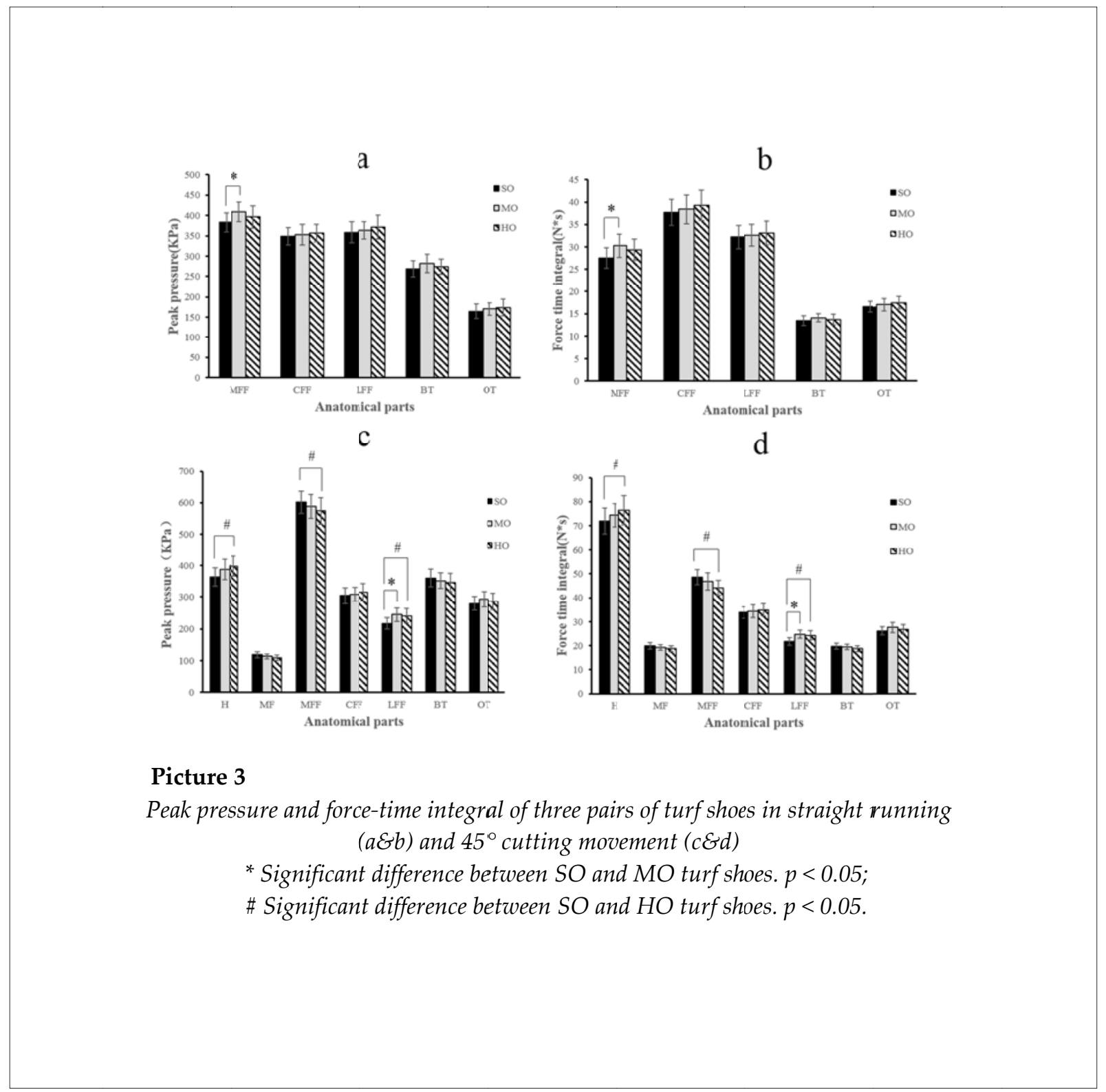

\section{Discussion}

In the present study, kinematic and kinetic data of soccer players were collected in laboratory conditions during straight running and cutting on an artificial turf surface when wearing turf shoes of three different degrees of outsole hardness. In this study we obtained biomechanical data of soccer related movements leading to the better understanding of playershoe-surface interaction considering shoes of different outsole hardness.

It was hypothesized that greater peak vertical GRF and a higher VALR would be generated when wearing shoes with $\mathrm{HO}$ compared to $\mathrm{MO}$ and $\mathrm{SO}$ during cutting movement. However, the results of this study showed no significant differences in peak vertical GRF between different shoes conditions. However, the VALR in the $\mathrm{HO}$ condition was significantly higher compared to the SO condition. The higher VALR when wearing shoes with $\mathrm{HO}$ may increase impact forces acting on lower limbs and lead to potential risks of tibial stress fracture and plantar fasciitis (Aerts and Clercq, 1993; Zadpoor and Nikooyan, 2011). In this study, higher initial peak value was found in $\mathrm{HO}$ shoes compared to shoes with SO and MO. Previous research showedl no difference in vertical impact forces between different hardness of 
outsoles (Nigg et al., 2012). That finding was consistent with the results of the study of Cheung et al. (2008), which showed a shorter time to the initial vertical force peak when wearing shoes with hard outsoles, resulting in a higher loading rate. That hard outsole will speed up the loading rate of approaching initial vertical force peak. Stacoff et al. (1988) developed a biomechanical model and observed that hard soles increased the lever arm of the subtalar joint axis, generating a faster initial eversion movement. This could explain similar impact force peaks for hard and soft outsoles. The Soft Outsole (SO) (E = 5.92 Mpa) under stress would produce more compression deformation compared to $\mathrm{MO}(\mathrm{E}=7.21 \mathrm{Mpa})$ and $\mathrm{HO}(\mathrm{E}=9.68 \mathrm{Mpa}$ ) (Li et al., 2015). An additional deceleration distance is created during the first 25 ms of contact time. This mechanism of passive shock attenuation could compensate for the difference in outsole hardness.

Soccer players frequently perform cutting movement, involving a rapid change of heading direction by a sudden and forceful twist of the lower limb with the flexed knee while the foot is being planted on the surface (Zarins et al., 1983). During these movements, the lower limb joints are subjected to substantial kinetic and kinematic changes compared with straight-ahead running, and these changes were implicated with noncontact anterior cruciate ligament (ACL) injuries (Besier et al., 2001). Questionnaires and video analyses have described medial collapse of the knee as a component of noncontact ACL injuries during cutting and landing maneuvers (Chaudhari and Andriacchi, 2006). This mechanism is supported by computer simulation studies, showing that large moments of the knee adductor increase the stress on ACL (Olsen et al., 2004).

Dynamic changes of direction have been determined as a risky factor of non-contact injuries in soccer. A 3-dimensional gait analysis was used in the dynamic setting. Lower limb biomechanical changes were identified during sidestep cutting maneuvers that may influence knee injuries (Besier et al., 2001; Mclean et al., 2003; Sun et al., 2017). During the stance phase of $45^{\circ}$ cut, decreased knee flexion angles of players wearing shoes with $\mathrm{HO}$ reduced the ability of impact absorption of their lower extremity, putting the knee at risk of injury (Derrick et al., 2002).
However, players wearing shoes with SO showed a significantly higher knee abductor angle than players wearing shoes with $\mathrm{HO}$ in the frontal plane. McLean et al. (2004) indicated an increased risk of ACL injury with increased knee abduction angles during movements involving rapid changes of direction. Another study found that while the mechanism of frontal plane loading during landing and cutting tasks was thought to differ, an increased knee valgus load during cutting constituted a risk factor of non-contact ACL injury (Besier et al., 2001). Current studies indicate that stiffer materials may reduce the motion of the foot at the frontal plane and reduce the impact on lower limbs. Sudden changes of heading directions while running increase forces acting on the lower limbs and this could result in non-contact musculoskeletal injuries (Schrier et al., 2014). Increased pressure and force-time integral in the heel region when wearing shoes with $\mathrm{HO}$ may result in a high potential risk of tibial stress fractures and plantar fasciitis (Lieberman et al., 2010). The results speculated that soft materials in the heel region of turf shoe outsoles may reduce the risk of non-contact injuries during soccer related movements.

Players wearing shoes with SO showed significant greater peak pressure and force-time integral in the MFF compared to players wearing shoes with $\mathrm{HO}$, also greater than when wearing shoes with $\mathrm{MO}$, although the difference was nonsignificant. During the stance phase of cutting movement, the medial forefoot bears more loads while wearing shoes with $\mathrm{SO}$ compared to other conditions. Greater pressure and force time integral would provide more propulsion to enhance athletic performance (Müller et al., 2010), but excessive pressure and an accumulative effect in a small area may lead to calluses in plantar skin, forefoot pain or even metatarsal stress fractures (Carlson et al., 2000; Grouios, 2004; Keijsers et al., 2013). This is consistent with the conclusion that higher forefoot pressure of the blade cleat design is substantially more harmful than in case of other cleats (Bentley et al., 2011). In contrast, players wearing shoes with SO showed significantly smaller peak pressure in the LFF than when wearing shoes with $\mathrm{MO}$ and $\mathrm{HO}$. Compared to the HO group, plantar pressure of MFF significantly increased when wearing shoes with SO, while plantar pressure in the LFF 
significantly decreased. Players wearing shoes with SO showed higher ankle dorsiflexion and inversion than when wearing shoes with $\mathrm{HO}$. During the stance of $45^{\circ}$ cut, soft outsoles were found to achieve higher maximum pronation, and this would result in more loading to the medial side of the ankle (De Wit et al., 1995). Compared with wearing shoes with $\mathrm{HO}$, the force-time integral in MFF was significantly higher when wearing shoes with SO. The increase of the forcetime integral in MFF when wearing shoes with SO may be due to the rise of pressure and accumulative contact time in this region. The landing pattern of $45^{\circ}$ cut was heel to toe with the LFF bearing passive force, thus soft materials $(\mathrm{E}=5.92 \mathrm{Mpa})$ of $\mathrm{SO}$ under stress might produce more compression deformation. During the stance phase of cutting movement, LFF and heel regions when wearing shoes with $\mathrm{HO}$ may bear more plantar pressure. The explanation is that softer material could function as a spring or cushion to relieve passive force, which may reduce noncontact injury risks on lower limbs.

There are several limitations of this study. Firstly, in this research only adult male soccer players in a no-fatigue state were studied. Results may not be representative of female players or different ages. Secondly, the experiment was performed in the laboratory conditions which differ from the soccer field environment. Thirdly, the artificial turf selected for this study without any infill was not in line with the standard of FIFA third-generation (3G) artificial turf, which may result in different loading mechanics. Lastly, the cutting direction in this study was anticipated. However, unanticipated cutting is more likely to represent the movements during a game situation and is considered to constitute an increased risk of injury compared to anticipated cutting. These factors should be taken into consideration in future research.

\section{Conclusion}

In summary, turf cleats shoes with different outsole hardness showed significant differences in kinematics and kinetics of lower limbs during straight running and cutting movements. During the stance phase of straight running, peak pressure and force-time integral in MFF of players wearing shoes with MO were significantly higher than in players wearing shoes with SO. During the stance phase of $45^{\circ}$ cut, players wearing shoes with SO showed increased knee flexion and knee abduction angles. The knee kinematics suggested that the ACL risk factors may be reduced in some cases when wearing shoes with $\mathrm{MO}$ and HO. A higher VALR and excessive plantar pressure in the heel $(\mathrm{H})$ of players wearing shoes with $\mathrm{HO}$ may increase the risk of tibia fractures, while higher plantar pressure in the MFF of players wearing shoes with SO may also cause calluses in plantar skin, forefoot pain or even metatarsal stress fractures. Results of this study provide scientific evidence and suggestions for the material selection of turf cleats shoes, indicating that softer material in the heel $(\mathrm{H})$ region and medium hardness material in the MFF region can improve sports performance and reduce the risk of non-contact injuries.

\section{Acknowledgements}

The study was sponsored by the National Natural Science Foundation of China (81301600), K.C.Wong Magna Fund in Ningbo University, and Zhejiang Xinmiao Innovation Talents Scheme (2015R405094).

\section{References}

Aerts P, Clercq DD. Deformation characteristics of the heel region of the shod foot during a simulated heel strike: the effect of varying midsole hardness. J Sports Sci, 1993; 11(5): 449-461

Bentley JA, Ramanathan AK, Arnold GP, Wang W, Abboud RJ. Harmful cleats of football boots: a biomechanical evaluation-foot and ankle surgery. Foot Ankle Surg, 2011; 17(3): 140-154

Besier TF, Lloyd DG, Cochrane JL, Ackland TR. External loading of the knee joint during running and 
cutting maneuvers. Med Sci Sports Exerc, 2001; 33(7): 1168-1175

Chaudhari AM, Andriacchi TP. The mechanical consequences of dynamic frontal plane limb alignment for non-contact ACL injury. J Biomech, 2006; 39(2): 330-338

Cheung RT, Ng GY. Influence of different footwear on force of landing during running. Phys Ther, 2008; 88(5): 620-628

Clarke TE, Frederick EC, Harnill CL. The effects of shoe design parameters on rearfoot control in running. Med Sci Sports Exerc, 1983; 15(5): 376-381

De Wit B, De Clercq D, Lenoir M. The effect of varying midsole hardness on impact forces and foot motion during foot contact in running. J Appl Biomech, 1995; 11: 395-406

Eils E, Streyl M, Linnenbecker S, Thorwesten L, Völker K, Rosenbaum D. Characteristic plantar pressure distribution patterns during soccer-specific movements. Am J Sports Med, 2004; 32(1): 140-145

Grouios G. Corns and calluses in athletes' feet: a cause for concern. The Foot, 2004; 14(4): 175-184

Hennig EM. Plantar pressure measurements for the evaluation of shoe comfort, overuse injuries and performance in soccer. Footwear Sci, 2014; 6(2): 119-127

Hennig EM. The influence of soccer shoe design on player performance and injuries. Res Sports Med, 2011; 19(3): 186-201

Hennig EM, Milani TL, Lafortune MA. The use of ground reaction force parameters for the prediction of peak tibial accelerations in running with different footwear. J Appl Biomech, 1993; 9(4): 306-314

Keijsers NLW, Stolwijk NM, Louwerens JWK, Duysens J. Classification of forefoot pain based on plantar pressure measurements. Clin Biomech, 2013; 28(3): 350-356

Lieberman DE, Venkadesan M, Werbel WA, Daoud AI, D'Andrea S, Davis IS, Pitsiladis Y. Foot strike patterns and collision forces in habitually barefoot versus shod runners. Nat, 2010; 463(7280): 531-535

Li F, Mei Q, Gu Y. Effects of unstable elements with different hardness on lower limb loading. Acta Bioeng Biomech, 2015; 17(2): 85-92

Matsuo K, Moriyasu K, Nishiwaki T. Basic study on cleat shape design in soccer boots. Footwear Sci, 2011; 3(1): 101-102

Mclean SG, Huang X, Su A, Bogert AJVD. Sagittal plane biomechanics cannot injure the ACL during sidestep cutting. Clin Biomech, 2004; 19(8): 828-838

McLean SG, Su A, van den Bogert AJ. Development and validation of a 3-D model to predict knee joint loading during dynamic movement. J Biomech Eng, 2003; 125(6): 864-874

Morio C, Lake MJ, Gueguen N, Rao G, Baly L. The influence of footwear on foot motion during walking and running. J Biomech, 2009; 42(13): 2081-2088

Müller C, Sterzing T, Lange J, Milani TL. Comprehensive evaluation of player-surface interaction on artificial soccer turf. Sports Biomech, 2010; 9(3): 193-205

Nigg BM, Bahlsen HA, Luethi SM, Stoke S. The influence of running velocity and midsole hardness on external impact forces in heel-toe running. J Biomech, 1987; 20: 951-959

Nigg BM, Baltich JM, Maurer C, Federolf P. Shoe midsole hardness, sex and age effects on lower extremity kinematics during running. J Biomech, 2012; 45(9): 1692-1697

Nigg BM, Cole GK, Bruggemann GP. Impact forces during heel-to-toe running. J App Biomech, 1995; 11: 407432

Olsen OE, Myklebust G, Engebretsen L, Bahr R. Injury mechanisms for anterior cruciate ligament injuries in team handball a systematic video analysis. Am J Sport Med, 2004; 32(4): 1002-1012

Sakaguchi M, Wannop J, Madden R, Koska D, Stefanyshyn D. Effects of shoe bending stiffness and surface stiffness on lower extremity biomechanics during running. Footwear Sci, 2015; 7(1): 4-6

Stacoff A, Denoth J, Kaelin X, Stuessi E. Running injuries and shoe construction: Some possible relationships. Int J Sport Biomech, 1988; 4: 342-357 
Sterzing T, Schweiger V, Ding R, Cheung JT, Brauner T. Influence of rear foot and forefoot midsole hardness on biomechanical and perception variables during heel-toe running. Footwear Sci, 2013; 5(2): 71-79

Schrier NM, Wannop JW, Lewinson RT, Worobets J, Stefanyshyn D. Shoe traction and surface compliance affect performance of soccer-related movements. Footwear Sci, 2014; 6(2): 69-80

Sun D, Gu YD, Mei QC, Baker JS. Different soccer stud configurations effect on running and cutting movements. Int. J. of Biomedical Engineering and Technology, 2017; 24(1): 19-32

Wang SW, Wang WD, Zhou DM. Rubber joint for fatigue crack factors Numerical Analysis of Certain Vehicle. App Mech Mater, 2011; 66: 1505-1508

Wong PL, Chamari K, Mao DW, Wisløff U, Hong Y. Higher plantar pressure on the medial side in four soccer-related movements. Br J Sports Med, 2007; 41(2): 93-100

Zadpoor AA, Nikooyan AA. The relationship between lower-extremity stress fractures and the ground reaction force: a systematic review. Clin Biomech, 2011; 26(1): 23-28

Zarins B, Rowe CR, Harris BA, Watkins MP. Rotational motion of the knee. Am J Sports Med, 1983; 11(3): 152156

Zhao H, Allanson D, Ren XJ. Use of Shore Hardness Tests for In-Process Properties Estimation/Monitoring of Silicone Rubbers. J Mater Sci Chem Eng, 2015; 3(7): 142-147

\section{Corresponding author:}

Yaodong Gu,

Faculty of Sports Science, Ningbo University, China

No.818 Fenghua road, Ningbo China 315211

Tel: +86-574-87609376; Fax: +86-574-87600456

Email: guyaodong@hotmail.com 